\title{
Hybrid Procedure Utilizing Stent Grafts to Stabilize Distal Flaps after Common and Superficial Femoral Endarterectomy
}

\author{
David V. Pham1, Bogdan Protyniak1, Samuel Hui', Ryan N. Cappa², \\ George Constantinopoulos ${ }^{1}$ \\ ${ }^{1}$ Department of Surgery, Monmouth Medical Center, Long Branch, USA \\ ${ }^{2}$ School of Medicine, St. George's University, St. George's, Grenada \\ Email: Protyniak@gmail.com
}

Received 9 February 2015; accepted 6 March 2015; published 9 March 2015

Copyright (C) 2015 by authors and Scientific Research Publishing Inc.

This work is licensed under the Creative Commons Attribution International License (CC BY). http://creativecommons.org/licenses/by/4.0/

c) $\underset{\mathrm{EY}}{\mathrm{O}}$ Open Access

\begin{abstract}
Background: Endarterectomy has long been the standard for common femoral artery (CFA) occlusive disease. Hybrid procedures utilizing endovascular and open techniques have recently been used for revascularization. The purpose of this study was to evaluate the effectiveness of the use of a stent graft to stabilize the distal flap and prevent further dissection after extensive endarterectomy. Methods: All patients from Monmouth Medical Center in Long Branch, NJ from September 2008 to March 2013 who underwent an extensive common and superficial femoral (SFA) endarterectomy combined with the use of a Viabahn (Gore Medical) stent graft to stabilize the distal flap were included in the study. These stents were deployed in the proximal SFA after extensive endarterectomy, under direct visualization, without the aid of fluoroscopy. Due to the location in the SFA, these flaps were not amenable to suture tacking. Results: Fifteen patients met these criteria and were included in our study. Twelve patients underwent femoral endarterectomy for severe claudication and three patients for limb salvage. Technical success was achieved in all 15 patients. Five patients also had stents placed proximally to increase inflow and one patient had an additional stent placed distally to improve outflow. There were no intraoperative or postoperative complications. Conclusion: Stent graft placement allows a more extensive endarterectomy to be performed by stabilizing the distal flap allowing a safe transition into the true lumen that is not possible with suture tacking.
\end{abstract}

\section{Keywords}

Common Femoral Endarterectomy, Superficial Femoral Endarterectomy, Stent Graft, Distal Flap, Vollmar Ring Dissector, Viabahn Stent 


\section{Introduction}

Patients with peripheral arterial disease (PAD) often have multifocal atherosclerotic lesions and substantial comorbidities that increase their operative risk. Limb revascularization previously required lengthy and sometimes staged procedures that were associated with increased morbidity and mortality. The advent of endovascular techniques changed the management of many PAD lesions.

Multiple studies have demonstrated the benefits of endovascular therapy for superficial femoral artery (SFA) occlusive disease [1]-[7]. However, unlike superficial femoral artery atherosclerotic disease, lesions in the common femoral artery (CFA) require special consideration with regard to endovascular treatment. Studies utilizing endovascular therapy for CFA occlusive disease have demonstrated variable results [8]-[11]. The anatomical location of CFA atherosclerotic disease precludes sole endovascular therapy. Lesions are often bulky, eccentric, and located at branch points, preventing ipsilateral access and stent deployment [12]. Stent placement across the inguinal ligament can lead to stent fracture and occlusion from intimal hyperplasia [13]. Furthermore, stent placement for CFA disease can sacrifice collaterals provided by the profunda and complicate future surgeries [13]. Studies have demonstrated that endarterectomy remains the standard of care for CFA occlusive disease [13] [14].

Recently, hybrid techniques for multifocal atherosclerotic disease have gained popularity [15]-[24]. These procedures use the proven durability of common femoral endarterectomy with concomitant endovascular management for both inflow and outflow lesions. As a result, multivessel disease can be treated in one procedure, without major hemodynamic changes, minimal blood loss, and limited risk of perioperative complications [16].

One limitation of common femoral endarterectomy is the ability to tack down unsafe distal flaps. With severe atherosclerotic disease, an incomplete endarterectomy may be performed for fear that the flap may be located too far distally to be safely tacked down with sutures, or a longer arteriotomy has to be made in order to create a safe endpoint for the endarterectomy. In our study, we employed a hybrid technique utilizing stent grafts to stabilize the distal flap in a location not amenable to suture tacking, allowing a more extensive common femoral and superficial femoral endarterectomy to be performed.

\section{Methods}

All patients at Monmouth Medical Center in Long Branch, NJ that underwent a femoral endarterectomy combined with the placement of a stent to stabilize the distal flap from September 2008 to March 2013 were included in this study. Procedures with stent placement not used to stabilize the distal flap were not included. The age, sex, type of stent, presenting complaints, and complications were evaluated.

\section{Surgical Technique}

Isolation of the common femoral, superficial femoral, and profunda arteries was first carried out. A guidewire was placed distally and kept there throughout the procedure (Figure 1). Remote endarterectomy was then carried out using a Vollmar Ring Dissector [LeMaitre Vascular] (Figure 2). A Viabahn stent (GORE VIABAHN

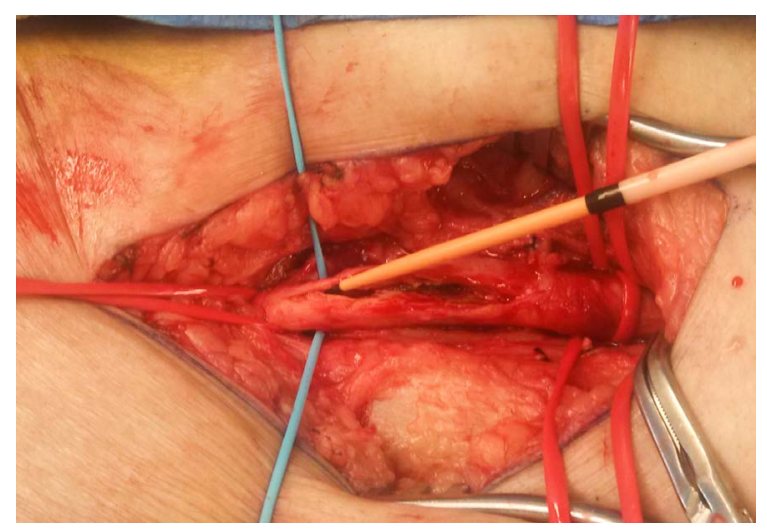

Figure 1. Exposure and arteriotomy of the CFA with guidewire placement. 


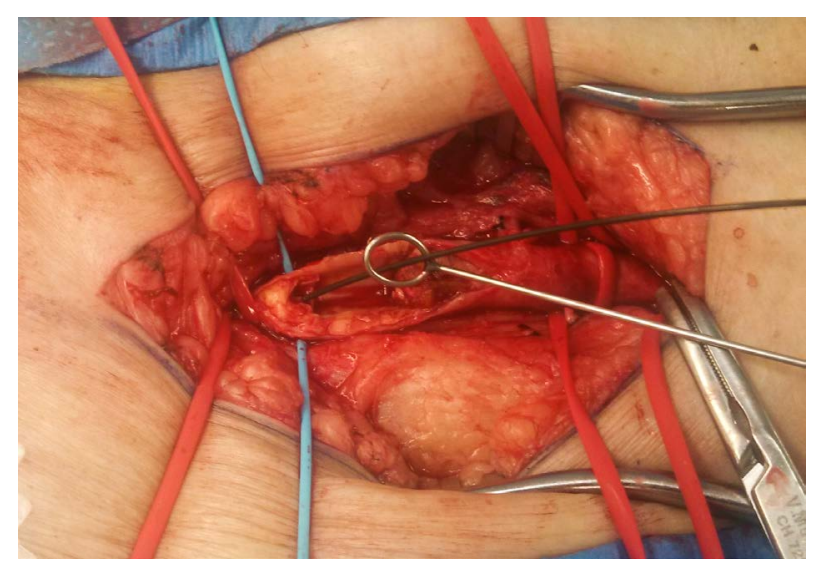

Figure 2. Vollmar ring dissector for distal remote endarterectomy.

Endoprosthesis, expanded polytetrafluoroethylene (ePTFE) liner attached to an external nitinol stent) was then deployed in the proximal SFA, under direct supervision, without the aid of fluoroscopy. The proximal end of the stent is positioned to be deployed at the distal end of the arteriotomy (Figure 3). After completion of the endarterectomy (Figure 4), a Bovine pericardium patch was then used to repair the arteriotomy (Figure 5). A completion arteriogram was then performed in cases where fluoroscopy was needed for treatment of other lesions (Figure 6).

\section{Results}

There were a total of 15 patients that underwent femoral endarterectomy with distal flap stent placement. Twelve patients underwent endarterectomy for debilitating claudication and three for limb salvage. The average age was 75 years. Sixty percent were female. Five patients had an additional stent placed proximally in the external iliac artery to increase inflow. One patient had an additional stent placed distally to increase outflow. Technical success was achieved in all patients without any intraoperative or postoperative complications.

\section{Discussion}

Endarterectomy has long been the standard of care for common femoral artery occlusive disease, achieving over 90\% immediate technical success rate [13] [14]. Many sources have demonstrated long term results for common femoral endarterectomy [13] [14] [25]. Ballotta et al. demonstrated in a 7-year study that the primary patency, primary-assisted patency, and limb salvage rates after CFA endarterectomy was $96 \%, 100 \%$, and $100 \%$, respectively [14]. Kang et al. also reported over $90 \%$ patency rates at a mean follow up of 27 months [13]. These authors concluded that endarterectomy should remain the standard of care for CFA occlusive disease [13] [14].

Recent studies involving solely endovascular treatment for CFA lesions are conflicting [8]-[11]. Bonvini et al. analyzed 360 consecutive percutaneous interventions of the CFA for atherosclerotic disease [8]. Balloon angioplasty was performed as the primary intervention in virtually all cases, while $36.9 \%$ of procedures required a stent for suboptimal angioplasty [9]. Angiography demonstrated $>30 \%$ residual post-procedure stenosis in $7.2 \%$ [9]. Medium-term follow-up at one year showed restenosis $>50 \%$ in $27.6 \%$ and target lesions revascularization in $19.9 \%$ [9]. Soga et al. evaluated endovascular therapy for CFA and demonstrated a 1- and 5-year primary patency rate of $73.4 \%$ and $46.9 \%$, respectively [10]. Dattilo et al. showed more promising results in their study of 31 CFA endovascular procedures, with technically success in $90 \%$ of cases and an overall 1-year primary and secondary patency rate of $88 \%$ and $92 \%$, respectively [11].

Despite the somewhat successful short- and medium-term results of endovascular therapy, there are many surgeons who are opposed to the procedure. Common femoral artery stenting prevents future endovascular access, makes distal bypass surgery difficult, and may sacrifice collaterals from the profunda artery [13] [14]. Furthermore, placing a stent across a joint or the inguinal ligament predisposes it to fracture and intimal hyperplasia [13] [14] [18]. 


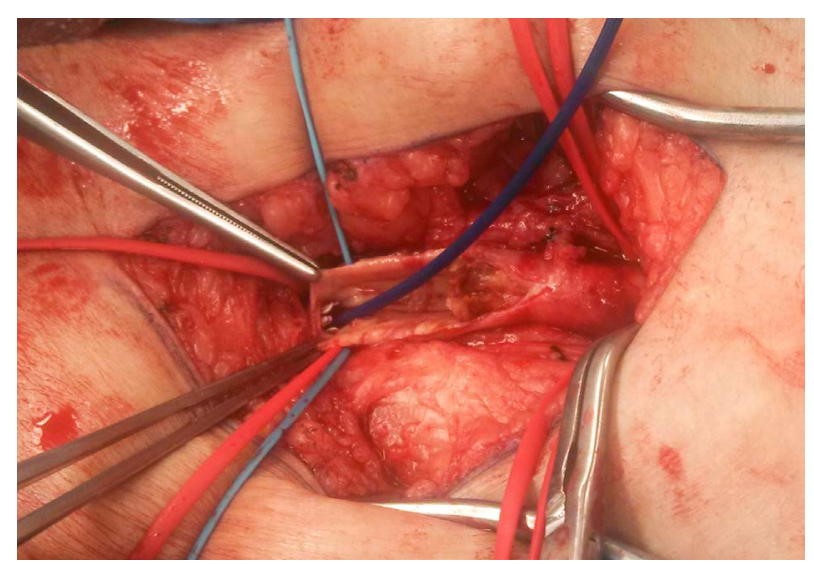

Figure 3. Deployment of a Viabahn stent under direct visualization.

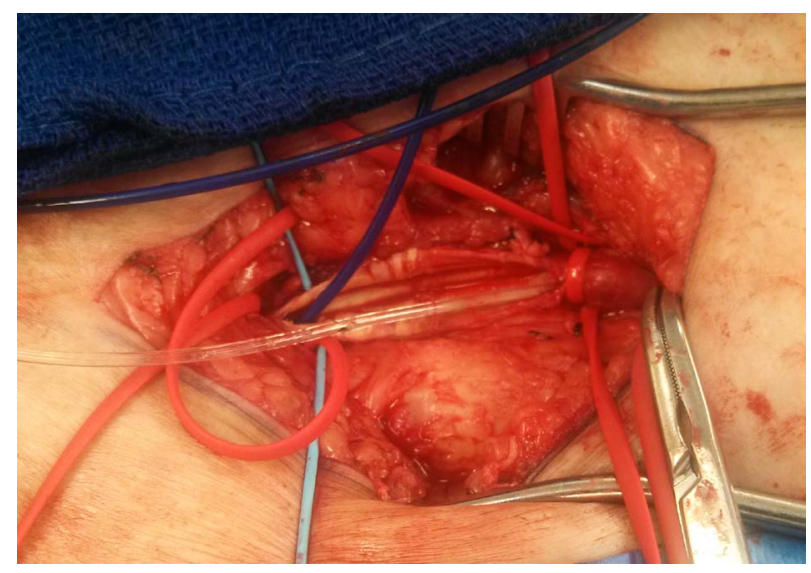

Figure 4. Completed endarterectomy.

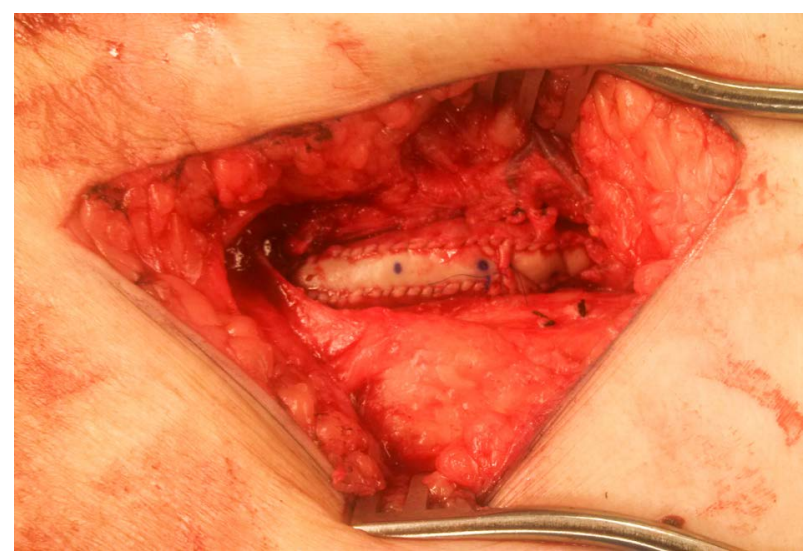

Figure 5. Repair of the arteriotomy using a Bovine pericardium patch.

The recent evolution of hybrid procedures, utilizing open and endovascular techniques, obviates the risks associated with CFA stenting and has gained increasing popularity in treating multifocal disease [15]-[24]. One advantage of the hybrid procedure is the ability to treat more complex anatomy using less invasive procedures in patients considered high medical risk [16]. Patent inflow and outflow can be achieved with endovascular techniques through the same arteriotomy, using limited surgical exposure. This can also be done under local anesthesia 


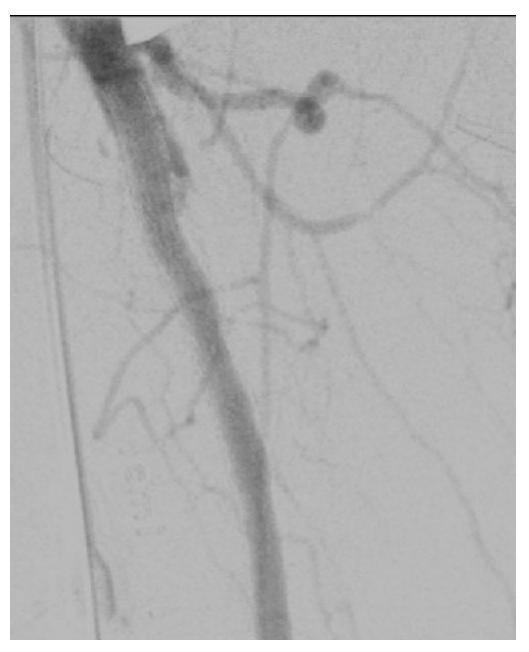

Figure 6. Completion arteriogram.

for high risk patients. Furthermore, with limited exposure, there is a decreased risk of an infection. Many sources have reported on the results of hybrid procedures in the treatment of common femoral artery occlusive disease. Chang et al. presented 171 patients who underwent common femoral endarterectomy combined with the placement of iliac stents or stent grafts with 5-year primary, primary assisted, and secondary patencies of $60 \%$, $97 \%$, and $98 \%$, respectively [17]. Hayes et al. combined femoral endarterectomy with distal femoral-popliteal stent graft placement to avoid traditional femoral-popliteal bypass [18]. Piazza et al. evaluated patients with extensive iliac and common femoral occlusive disease by comparing open reconstruction to a hybrid approach [19]. They reported similar short- and long-term efficacy with the hybrid technique and recommended this approach in high-risk patients [19].

In our study, we employed a hybrid technique that allowed a more extensive endarterectomy to be performed, compared to conventional endarterectomy, while still maintaining safety by deploying a stent graft to stabilize the distal flap. Traditionally, the distal flaps are tacked down using sutures to prevent further dissection [12]. However, in most patients the disease progresses into the SFA [14] with no clear transition point, leaving a distal flap that is difficult to safely tack down. Feng et al. reported the largest study of 104 cases of common femoral endarterectomy combined with SFA stenting for chronic lower extremity ischemia over three years [12]. The authors performed an endarterectomy, tacked the distal flap using sutures, and then placed a stent proximal to the distal endarterectomy edge to avoid an intimal edge flap [12]. We were able to perform a more extensive endarterectomy using the Vollmar Ring Dissector (LeMaitre Vascular) to the point where the distal flap was not visualized in the SFA and, therefore, not amenable to suture tacking. Use of a stent graft at this location provided a safe transition to the true lumen not possible with suture tacking. Furthermore, the stent graft was deployed under direct visualization without the need for fluoroscopy.

This study has several limitations. The data used in this study was retrospectively collected from a single institution, resulting in a small number of cases. Second, this study did not assess for long-term efficacy results. Despite these limitations, our study describes a novel hybrid technique where a more extensive CFA endarterectomy can be performed using the Vollmar Ring Dissector with distal flap stabilization via stenting.

\section{Conclusion}

Endarterectomy is still the standard of care for common femoral artery occlusive disease. We employed a hybrid technique where a stent graft was used to stabilize the distal flap. Stent graft placement allows a more extensive endarterectomy to be performed by stabilizing the distal flap allowing a safe transition into the true lumen that is not possible with suture tacking.

\section{References}

[1] Chalmers, N., Walker, P.T., Belli, A.M., Thorpe, A.P., Sidhu, P.S., Robinson, G., et al. (2013) Randomized Trial of the SMART Stent versus Balloon Angioplasty in Long Superficial Femoral Artery Lesions: The SUPER Study. Cardi- 
ovascular and Interventional Radiology, 36, 353-361. http://dx.doi.org/10.1007/s00270-012-0492-Z

[2] Sakamoto, Y., Hirano, K., Iida, O., Soga, Y., Suzuki, K., Muramatsu, T., et al. (2013) Five-Year Outcomes of SelfExpanding Nitinol Stent Implantation for Chronic Total Occlusion of the Superficial Femoral and Proximal Popliteal Artery. Catheterization and Cardiovascular Interventions, 82, 251-256. http://dx.doi.org/10.1002/ccd.24935

[3] Gabrielli, R., Rosati, M.S., Vitale, S., Baciarello, G., Siani, A., Chiappa, R., et al. (2012) Randomized Controlled Trial of Remote Endarterectomy versus Endovascular Intervention for TransAtlantic Inter-Society Consensus II D Femoropopliteal Lesions. Journal of Vascular Surgery, 56, 1598-1605. http://dx.doi.org/10.1016/j.jvs.2012.06.081

[4] Bakoyiannis, C.N., Tsekouras, N., Matthaiou, A., Georgopoulos, S.E., Econopoulos, K.P., Tsigris, C., et al. (2009) Guided Remote Endarterectomy for Superficial Femoral Artery Occlusions: A Technical Note. International Angiology, 28, 380-384.

[5] Antoniou, G.A., Koutsias, S., Antoniou, S.A. and Giannoukas, A.D. (2008) Remote Endarterectomy for Long Segment Superficial Femoral Artery Occlusive Disease. A Systematic Review. European Journal of Vascular and Endovascular Surgery, 36, 310-318. http://dx.doi.org/10.1016/j.ejvs.2008.04.005

[6] Ali, A.T., Kalapatapu, V., Ahmed, O., Moursi, M. and Eidt, J.F. (2007) Remote Superficial Femoral Artery Endarterectomy: Early Results for TASC D Lesions in Patients with Severe Ischemia. Vascular and Endovascular Surgery, 41, 310-315. http://dx.doi.org/10.1177/1538574407302751

[7] Martin, J.D., Hupp, J.A., Peeler, M.O. and Warble, P.O. (2006) Remote Endarterectomy: Lessons Learned after More than 100 Cases. Journal of Vascular Surgery, 43, 320-326. http://dx.doi.org/10.1016/j.jvs.2005.10.017

[8] Bonvini, R.F., Rastan, A., Sixt, S., Beschorner, U., Noory, E., Schwarz, T., et al. (2013) Angioplasty and Provisional Stent Treatment of Common Femoral Artery Lesions. Journal of Vascular and Interventional Radiology, 24, 175-183. http://dx.doi.org/10.1016/j.jvir.2012.10.020

[9] Bonvini, R.F., Rastan, A., Sixt, S., Noory, E., Schwarz, T., Frank, U., et al. (2011) Endovascular Treatment of Common Femoral Artery Disease: Medium-Term Outcomes of 360 Consecutive Procedures. Journal of the American College of Cardiology, 58, 792-798. http://dx.doi.org/10.1016/j.jacc.2011.01.070

[10] Soga, Y., Tomoi, Y., Sato, K., Iida, O. and Yokoi, H. (2013) Clinical Outcome after Endovascular Treatment for Isolated Common Femoral and Popliteal Artery Disease. Cardiovascular Intervention and Therapeutics, 28, 250-257. http://dx.doi.org/10.1007/s12928-013-0164-1

[11] Dattilo, P.B., Tsai, T.T., Rogers, R.K. and Casserly, I.P. (2013) Acute and Medium-Term Outcomes of Endovascular Therapy of Obstructive Disease of Diverse Etiology of the Common Femoral Artery. Catheterization and Cardiovascular Interventions, 81, 1013-1022. http://dx.doi.org/10.1002/ccd.24475

[12] Feng, H., Chen, X.M., Li, C.Y., Zhu, R.M., Fang, J. and Wang, T.Y. (2012) Combined Common Femoral Artery Endarterectomy with Superficial Femoral Artery Stenting plus Shuxuening Injection Infusion for Chronic Lower Extremity Ischemia: 3-Year Results. Journal of Integrative Medicine, 18, 417-422.

[13] Kang, J.L., Patel, V.I., Conrad, M.F., Lamuraglia, G.M., Chung, T.K. and Cambria, R.P. (2008) Common Femoral Artery Occlusive Disease: Contemporary Results Following Surgical Endarterectomy. Journal of Vascular Surgery, 48, 872-877. http://dx.doi.org/10.1016/j.jvs.2008.05.025

[14] Ballotta, E., Gruppo, M., Mazzalai, F. and Da Giau, G. (2010) Common Femoral Artery Endarterectomy for Occlusive Disease: An 8-Year Single-Center Prospective Study. Surgery, 147, 268-274. http://dx.doi.org/10.1016/j.surg.2009.08.004

[15] Zou, J., Xia, Y., Yang, H., Ma, H. and Zhang, X. (2012) Hybrid Endarterectomy and Endovascular Therapy in Multilevel Lower Extremity Arterial Disease Involving the Femoral Artery Bifurcation. International Surgery, 97, 56-64. http://dx.doi.org/10.9738/0020-8868-97.1.56

[16] Matsagkas, M., Kouvelos, G., Amaoutoglou, E., Papa, N., Labropoulos, N. and Tassiopoulos, A. (2011) Hybrid Procedures for Patients with Critical Limb Ischemia and Severe Common Femoral Artery Atherosclerosis. Annals of Vascular Surgery, 25, 1063-1069. http://dx.doi.org/10.1016/j.avsg.2011.07.010

[17] Chang, R.W., Goodney, P.P., Baek, J.H., Nolan, B.W., Rzucidlo, E.M. and Powell, R.J. (2008) Long-Term Results of Combined Common Femoral Endarterectomy and Iliac Stenting/Stent Grafting for Occlusive Disease. Journal of Vascular Surgery, 48, 362-367. http://dx.doi.org/10.1016/j.jvs.2008.03.042

[18] Hayes Jr., D.J., Dougherty, M.J. and Calligaro, K.D. (2011) Management of Flush Superficial Femoral Artery Occlusions with Combined Open Femoral Endarterectomy and Endovascular Femoral-Popliteal Angioplasty and StentGrafting. Annals of Vascular Surgery, 25, 559.e19-559.e23.

[19] Piazza, M., Ricotta II, J.J., Bower, T.C., Kalra, M., Cuncan, A.A., Cha, S., et al. (2011) Iliac Artery Stenting Combined with Open Femoral Endarterectomy Is as Effective as Open Surgical Reconstruction for Severe Iliac and Common Femoral Occlusive Disease. Journal of Vascular Surgery, 54, 402-411. http://dx.doi.org/10.1016/j.jvs.2011.01.027

[20] Rosenthal, D., Martin, J.D., Schubart, P.J., Wellons, E.D., Shuler, F.W. and Levitt, A.B. (2004) Remote Superficial 
Femoral Artery Endarterectomy and Distal aSpire Stenting: Mid-Term Results. Journal of Vascular Surgery, 40, 67-72. http://dx.doi.org/10.1016/j.jvs.2004.03.038

[21] Rosenthal, D., Martin, J.D., Smeets, L., Devries, J.P., Gisbertz, S., Wellons, E.D., et al. (2006) Remote Superficial Femoral Artery Endarterectomy and Distal aSpire Stenting: Results of a Multinational Study at Three-Year Follow-Up. Journal of Cardiovascular Surgery, 47, 385-391.

[22] Aho, P.S. and Venermo, M. (2012) Hybrid Procedures as a Novel Technique in the Treatment of Critical Limb Ischemia. Scandinavian Journal of Surgery, 101, 107-113. http://dx.doi.org/10.1177/145749691210100206

[23] Nelson, P.R., Powell, R.J., Schermerhorn, M.L., Fillinger, M.F., Zwolak, R.M., Walsh, D.B., et al. (2002) Early Results of External Iliac Artery Stenting Combined with Common Femoral Artery Endarterectomy. Journal of Vascular Surgery, 35, 1107-1113. http://dx.doi.org/10.1067/mva.2002.124374

[24] Dosluoglu, H.H., Lall, P., Cherr, G.S., Harris, L.M. and Dryjski, M.L. (2010) Role of Simple and Complex Hybrid Revascularization Procedures for Symptomatic Lower Extremity Occlusive Disease. Journal of Vascular Surgery, 51, 1425-1435. http://dx.doi.org/10.1016/j.jvs.2010.01.092

[25] Cardon, A., Aillet, S., Jarno, P., Bensalah, K., Le Du, J., Idrissi, A., et al. (2001) Endarteriectomy of the Femoral Tripod: Long-Term Results and Analysis of Failure Factors. Annales de Chirurgie, 126, 777-782. http://dx.doi.org/10.1016/S0003-3944(01)00593-4 